\title{
Enhancing Pharmacovigilance Capabilities in the EU Regulatory Network: The SCOPE Joint Action
}

\author{
Anna Radecka ${ }^{1}$ - Louise Loughlin ${ }^{1} \cdot$ Mick Foy $^{1}$ - Margarida Viana de Ferraz Guimaraes ${ }^{2}$. Viola Macolic Sarinic ${ }^{3}$. \\ Marina Dimov Di Giusti ${ }^{4}$. Marina Lesicar ${ }^{4}$. Sabine Straus ${ }^{5}$. Dolores Montero ${ }^{6}$. Julia Pallos ${ }^{7}$. Jelena Ivanovic ${ }^{8}$. \\ June Raine ${ }^{1}$
}

Published online: 21 August 2018

(c) The Author(s) 2018

\begin{abstract}
In November 2013, a team of European regulators initiated the Strengthening Collaboration for Operating Pharmacovigilance in Europe (SCOPE) Joint Action. Funded by the Health Programme of the European Union, and with contributions from the involved Member States, SCOPE gathered information and expertise on how regulators in Member States run their national pharmacovigilance systems to meet the requirements of the pharmacovigilance legislation that came into effect in June 2012. The SCOPE project evaluated then-current practices and developed tools to further improve the skills and capability in the pharmacovigilance network. The project was divided into eight separate work streams, five of which concentrated on pharmacovigilance topics-collecting information on suspected adverse drug reactions, identifying and managing safety issues (signals), communicating risk and assessing risk minimisation measures, supported by effective quality management systems. The other three work streams focused on the functional aspects-coordination, communication and evaluation of the project. Through the project, SCOPE delivered guidance, training in key aspects of pharmacovigilance, and tools and templates to support best practice. The deliverables provide practical guidance that those working in the European national competent authorities can take to strengthen their national systems. The SCOPE outputs can be useful for other stakeholders involved in pharmacovigilance activities, including the pharmaceutical industry, healthcare professionals, patient and consumer organisations, and academia.
\end{abstract}

Anna Radecka

anna.radecka@mhra.gov.uk

1 Medicines and Healthcare products Regulatory Agency (MHRA), London, UK

2 INFARMED-National Authority of Medicines and Health Products, Lisbon, Portugal

3 World Health Organization (WHO), Geneva, Switzerland

4 Agency for Medicinal Products and Medical Devices of Croatia (HALMED), Zagreb, Croatia

5 Medicines Evaluation Board (MEB), Utrecht, The Netherlands

6 Agencia Española de Medicamentos y Productos Sanitarios (AEMPS), Madrid, Spain

7 National Institute of Pharmacy and Nutrition (OGYÉI), Budapest, Hungary

8 Italian Medicines Agency (AIFA), Rome, Italy

\section{Key Points}

The Strengthening Collaboration for Operating Pharmacovigilance in Europe (SCOPE) Joint Action brought together national medicines regulators from the European Economic Area to develop guidance, training in key aspects of pharmacovigilance, and tools and templates to support best practice.

The aim of the Joint Action was to strengthen the pharmacovigilance network in Europe and improve operating pharmacovigilance capabilities and collaborative working.

SCOPE training materials and outputs are publicly available for interested parties to use and implement according to their needs. 


\section{Introduction}

The Strengthening Collaboration for Operating Pharmacovigilance in Europe (SCOPE) Joint Action was a public initiative co-ordinated by the Medicines and Healthcare products Regulatory Agency (MHRA) in the United Kingdom (UK). The SCOPE Joint Action was created to support pharmacovigilance operations in the European network, minimise duplication of work, and make the best use of work sharing and resources. A key aim of the project was to help lower-resourced national competent authorities (NCAs) develop skills and capacity in pharmacovigilance, and thereby help safeguard public health in both national territories and the European Union (EU) as a whole.

The context of the SCOPE Joint Action arose from challenges for the EU pharmacovigilance network. First, in 2012 the burden of adverse drug reactions (ADRs) across EU Member States was estimated to cost healthcare systems a total of $€ 79$ billion per year. The societal burden is also significant, with 5\% of all hospital admissions thought to be due to an ADR, while ADRs are reported as the fifth most common cause of hospital death across the EU [1]. In the UK, approximately $6.5 \%$ of hospital admissions are caused by ADRs, at considerable cost on health and healthcare budgets [2]. Therefore, NCAs are required to continually adapt and update their processes to respond to and deal with these challenges.

Second, in July 2012, the new pharmacovigilance legislation specified in Regulation (EU) No 1235/2010 and Directive 2010/84/EU came into force across the EU, introducing new standards and requirements for all Member States to meet, including supporting the work of the Pharmacovigilance Risk Assessment Committee (PRAC). PRAC members are appointed by the EU Member States, and the committee includes independent experts appointed by the European Commission (EC), as well as representatives of healthcare professionals (HCPs) and patients [3].

The Joint Action aimed to provide NCAs with the knowledge, tools and confidence in their capability to identify and promote strengths and expertise while developing weaker pharmacovigilance areas in order to broaden capacity and breadth of work and meet their statutory obligations. The SCOPE outputs aim to provide practical advice and tools, supporting Good Vigilance Practice (GVP) guidelines and other existing systems.

The SCOPE Joint Action was funded by the Health Programme of the European Union 2008-2013, with contribution from the involved Member States, under Grant Agreement No. 20132102. The EC granted the SCOPE Joint Action $€ 3.3$ million, with a total budget of $€ 4.7$ when taking into account contributions from NCAs. Full details of the awarding agency, the Consumers, Health, Agriculture and Food Executive Agency (CHAFEA), can be found at http://ec.europa.eu/chafea/index.html.

\section{Planning of the Joint Action: Structure and Overview}

The SCOPE consortium consisted of 28 medicines regulators from the EU and European Economic Area (EEA), and was supported by several other organisations with specific interest and expertise in pharmacovigilance topics (Table 1). The outputs from the SCOPE Joint Action were developed through a number of work packages (WPs) (Fig. 1):

- Coordination (WP1) had overall responsibility for the coordination and project management of SCOPE, including financial management, reporting to the EC, and ensuring that the Joint Action was accomplished on time and with high-quality deliverables.

- Communication and dissemination (WP2) was created to effectively maintain internal communications between SCOPE partners, as well as disseminating information to external stakeholders.

- Evaluation (WP3) focused on the measurement and assessment of SCOPE activities and supported the achievement of the SCOPE objectives and outputs.

- ADR collection (WP4) focused on national schemes for the spontaneous reporting of ADRs by HCPs and patients, and aimed to provide NCAs with a better understanding of available systems and practices for collecting ADRs.

- Signal management (WP5) sought to further improve understanding of signal management within the EU NCA network, develop best practice in signal management, and provide training to national medicines regulators.

- Risk communication (WP6) focused on risk communication practices in the EU network, and aimed to understand communication channels, tools used, and effectiveness of different strategies and methods.

- Quality management systems (WP7) aimed to develop tools to support quality standards in pharmacovigilance systems, and to increase existing knowledge on quality management systems through provision of a training programme.

- Lifecycle Pharmacovigilance (WP8) explored existing standards for pharmacovigilance assessments and examined the availability and use of alternative data sources (outside of spontaneous reports) for pharmacovigilance assessments in different European NCAs.

The WPs were led by NCAs from seven Member States, and representatives from these organisations comprised the WP Leaders Group (Table 1), which was responsible for the 
Table 1 SCOPE Joint Action Partner NCAs and other organisations

Work package leaders

Agencia Española de Medicamentos y Productos Sanitarios (Spain), HALMED Agency for Medicinal Products and Medical Devices (Croatia), INFARMED National Authority of Medicines and Health Products (Portugal), Italian Medicines Agency (Italy), Medicines and Healthcare products Regulatory Agency (The United Kingdom), Medicines Evaluation Board (The Netherlands), National Institute of Nutrition (Hungary)

Project partners

Agence Nationale de Sécurité des Médicaments et des Produits de Santé (France), Agency for Medicinal Products and Medical Devices of the Republic of Slovenia (Slovenia), Bulgarian Drug Agency (Bulgaria), Danish Health Authority (Denmark), Federal Agency for Medicines and Health Products (Belgium), Finish Medicines Agency Fimea (Finland), Fundación Española para la Cooperación Internacional, Salud y Política Social (Spain), Health Products Regulatory Agency (Ireland), Icelandic Medicines Agency (Iceland), Medicines Authority (Malta), National Agency for Medicines and Medical Devices (Romania), National Organisation for Medicines (Greece), Norwegian Medicines Agency (Norway), Office for Registration of Medicinal Products, Medical Devices and Biocidal Products (Poland), Pharmaceutical Services of the Ministry of Health (Cyprus), State Agency of Medicines (Estonia), State Agency of Medicines of Latvia (Latvia), State Institute for Drug Control (Czech Republic), State Institute for Drug Control (Slovakia), State Medicines Control Agency (Lithuania), Swedish Medical Products Agency (Sweden)

General advisory board

European Medicines Agency (EMA), European Organisation for Rare Diseases (EURORDIS), International Society of Pharmacovigilance (ISoP), Uppsala Monitoring Centre (UMC)

Collaborating partners

Maastricht University, Netherlands Pharmacovigilance Centre Lareb, University Medical Centre Groningen (UMCG), University of Nottingham, Uppsala Monitoring Centre

NCA national competent authorities

Strategic level

Executive level

Implementation level

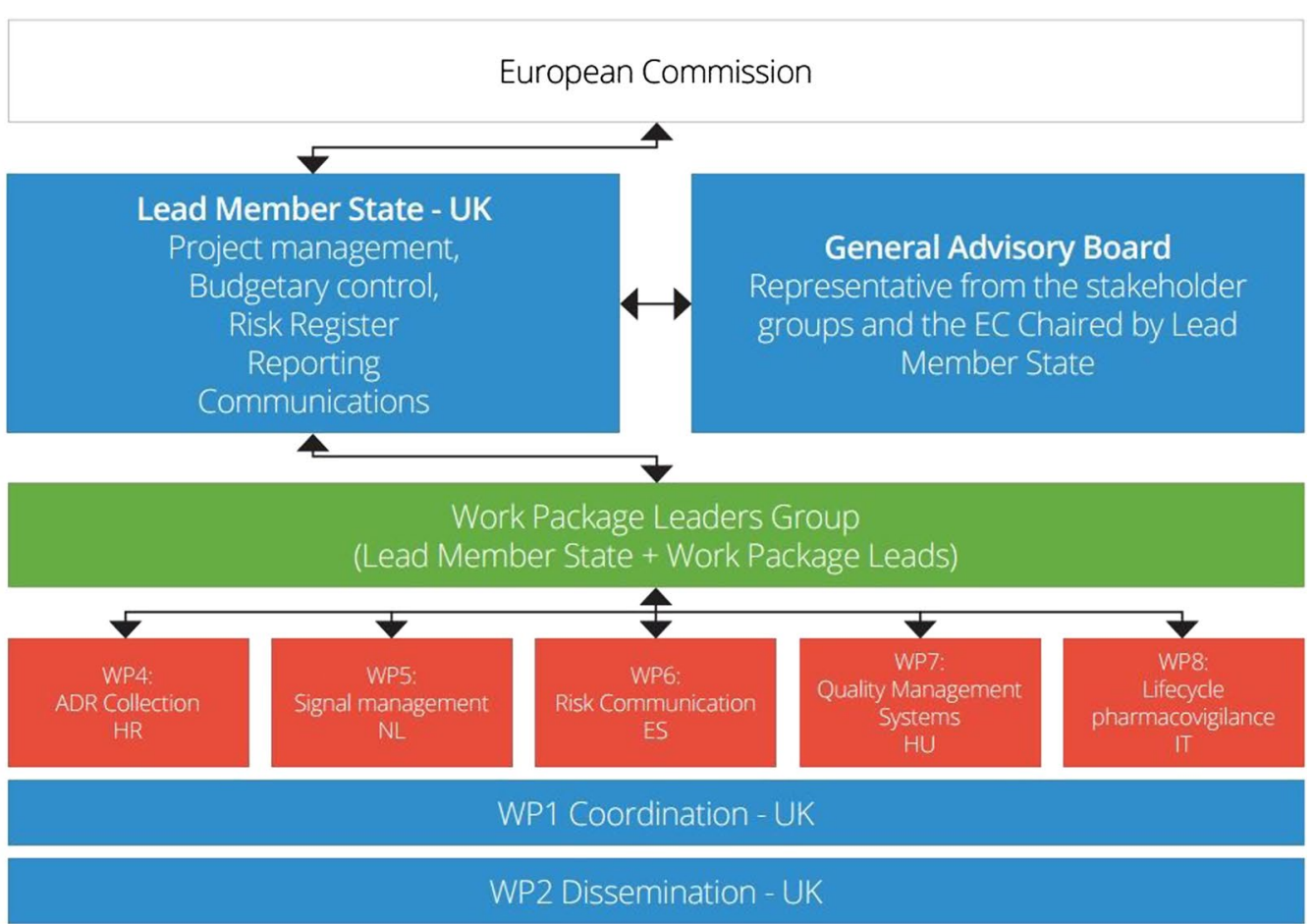

WP3 Evaluation - PT

Fig. 1 SCOPE Joint Action work packages and governance structure. SCOPE Strengthening Collaboration for Operating Pharmacovigilance in Europe, EC European Commission, $W P$ work package, $H R$ Croatia, $N L$ The Netherlands, ES Spain, HU Hungary, IT Italy, PT Portugal

delivery and management of SCOPE. The project was also supported by a General Advisory Board. The Board was formed of representatives from organisations independent of those delivering and managing the joint action, and provided strategic advice and enabled the delivery of high standard outputs and results (Table 1). 
This paper focuses on the work completed by the SCOPE consortium, and provides an overview of all outputs released to the pharmacovigilance network. The deliverables presented in this publication relate to the research conducted by the SCOPE Joint Action between 2013 and 2017. For a full description of the studies, please refer to the cited original survey reports published on the project website, or to scientific publications. The SCOPE outputs have been published on the project website (http://www.scopejointactio n.eu/), which will continue to be live until the end of 2019. These outputs will also be transferred to the CHAFEA and included in the project database. SCOPE learning materials were also transferred to the EU Network Training Centre (EU NTC), a joint training initiative endorsed by the Heads of Medicines Agencies (HMA) and the European Medicines Agency (EMA).

\section{Surveying the Landscape: Finding Out About Pharmacovigilance Practices}

In 2014, SCOPE work package members developed surveys aimed at identifying current pharmacovigilance practices of NCAs. These surveys focused on regulators' experience in the following areas: ADR collection, signal management, national methods of communication and web portals, quality management systems, and methods and processes for pharmacovigilance assessments. The questionnaires were distributed to 28 NCAs participating in the SCOPE Joint Action, as well as other non-partner organisations. A total of 764 questions were asked. National medicines regulators recognised the significance of the SCOPE research and provided a large amount of information about their national pharmacovigilance systems and strategies. Depending on the survey, between 25 and 28 NCAs returned questionnaires. As a result, these data provided a valuable source of information about current pharmacovigilance practices across the EU.

The results from all survey reports were used to inform SCOPE recommendations, training materials, guidance documents and other tools delivered over three years, as well as to design training sessions and workshops.

\subsection{Survey on Adverse Drug Reaction (ADR) Collection (WP4)}

An ADR is defined as a noxious and unintended response to a medicine, and the legislation requires that Member States take all measures to ensure that the ADR reporting legislative requirements are fully met [4]. Information about national pharmacovigilance systems, information technology (IT) system capabilities, implementation of patient reporting schemes, reporting forms in use, and electronic reporting developments was collected from national medicines regulators via web-based survey tools. In total, six questionnaires were developed and covered topics including national reporting systems, medication errors, patient reporting, awareness levels, reporting forms and IT systems and special report forms [5-7].

Overall, differences in national pharmacovigilance systems among NCAs were observed, including various systems for processing ADR reports; legal specifications in addition to the EU legal requirements; resources and budgets; and various IT systems. Therefore, recommendations from this work stream focused on different aspects of pharmacovigilance business activities using good practice examples from NCAs [5-7].

\subsection{Survey on Signal Management (WP5)}

The EU legislation introduced an EU-wide process for signal management, with specific responsibilities and interactions among all stakeholders involved [4]. A web-based survey was developed by WP5 to gather information on all aspects of signal management among the different Member States in the EU. The survey contained questions about general organisation, approaches to signal detection, signal validation and prioritisation, signal confirmation and assessment, and reports of special interest. The results outlined the existing heterogeneity in the process of signal management within the EU network. This heterogeneity appears to be beneficial for the whole system, especially with regard to the signal detection process. Several challenges were identified, such as the terminology and definitions used in the signal management process at Member State level, which lead to complexities in the interpretation and implementation of the existing legislation, the need for resources and good training, awareness of the available data sources and access to data, and the need for tools to support the signal management process [8].

\subsection{Surveys on National Methods of Communication and Web Portals (WP6)}

Two surveys were launched to gather information about current safety communication practices of NCAs. One survey explored existing methods of communication on safety of medicines developed by NCAs, and aimed to identify examples of good strategies, plans, and tools that could be considered. In parallel, the other survey focused on the use of web portals and asked about current practices related to the use of NCAs' websites and methods aiming to improve HCPs' awareness of safety information distributed by NCAs. The results from both surveys indicated that risk communication systems and processes are available in all NCAs, and that NCAs use similar methods for safety communication 
on medicines $[10,11]$. Strengths and limitations revealed by these surveys informed development of practical guidance on selected aspects of risk communication practice.

\subsection{Survey on Quality Management Systems (WP7)}

Three web-based questionnaires aimed at identifying quality management systems were developed, including processes and preferences. The first questionnaire asked Member States a series of questions on their national quality systems; the second questionnaire investigated how Member States approach resource management; and the third questionnaire focused on interactions between pharmacovigilance assessors and inspectors. Analysis of responses provided insight into the quality management practices of EU NCAs, including challenges that national medicines regulators are facing and good practices developed to operate their pharmacovigilance quality systems [12-14]. Survey results illustrated the diverse approaches used nationally, and informed development of a quality management toolkit that comprised of guidance documents, e-learning modules and other supporting tools (Table 2).

\subsection{Survey on Methods and Processes for Pharmacovigilance Assessments (WP8)}

Five questionnaires aimed to identify the current pharmacovigilance assessment methods and processes of NCAs, and contained questions about identification of the available data sources outside spontaneous reporting, risk management plan (RMP) assessments, post-authorisation safety and efficacy studies (PASS and PAES) protocols and study reports, benefit-risk assessment in the context of periodic safety update reports (PSURs) and referral procedures, and the relevant competency levels. The questionnaires reflected on the current assessment practice, with a focus on challenges, possible solutions and good practice examples. Survey analysis informed the development of recommendations, practical guides, and the design of a training programme (including e-learning). These tools can be used by NCAs to support the PRAC with high-quality assessment and advice on RMPs, PASS, PSURs and referral procedures [15].

\section{Using the Strengthening Collaboration for Operating Pharmacovigilance in Europe (SCOPE) Survey Results to Strengthen the Pharmacovigilance Network}

European NCAs differ in terms of structure, organisation and resources available. The SCOPE recommendations and outputs aim to complement the existing GVP guidance to enhance pharmacovigilance practice in the EU. The SCOPE project delivered sustainable outcomes for NCAs that last beyond the end of the project through the creation of training materials and living documents, which can be reviewed and adapted periodically. The SCOPE WPs focused on different aspects of pharmacovigilance practice and produced various deliverables depending on the network needs identified via SCOPE surveys. Thus, WPs delivered different numbers and types of outputs (Table 2).

\subsection{ADR Collection (WP4)}

\subsubsection{Overview of National ADR Reporting Schemes, Guidance Documents and e-Learning Modules}

A comprehensive overview of national ADR reporting schemes, presenting a variety of current methods and practices identified in WP4 surveys, were delivered in the form of three reports. These reports present an overview of national schemes and how ADR processes have been implemented across Europe.

Recommendations from this work, along with good practice examples identified in the WP4 surveys and via followup communication, informed the development of guidance documents, e-learning modules and other learning materials (Table 2). These tools are intended to support NCAs, including their regional centres and national pharmacovigilance centres where applicable, to strengthen their existing strategies and practice in order to increase the number and quality of suspected ADR reports.

\subsubsection{Development of a Web-Based ADR Reporting Form}

The 2012 pharmacovigilance legislation required all Member States to provide a web-based form for reporting suspected ADRs for patients and HCPs [4]. In 2014, the WP4 surveys found that ADRs can be spontaneously reported by patients to NCAs via the following channels: mail (24 NCAs), fax (20 NCAs), e-mail (21 NCAs), web-based forms (20 NCAs) and telephone calls (19 NCAs). One NCA also had mobile reporting availability. Of the Member States, 21 had a web form for receiving reports from HCPs. The same NCAs who did not have a web form for patients, also did not offer this way of reporting to HCPs. However, there were two exceptions; one NCA had a web form for HCPs, but not for patients, and another had a web-based application only for patients [5].

In $20 \%$ of NCAs, a web-based tool for ADR reporting was not made available, and some indicated interest in implementing a web form created by SCOPE via a follow-up questionnaire. Therefore, a web form was developed in the context of the SCOPE Joint Action to facilitate reporting by HCPs, patients, and their carers. 
Table 2 SCOPE Joint Action results and outputs, including e-learning modules

ADR collection (WP4)

Signal management (WP5)

Risk communications (WP6)

\section{ADR reporting}

An active approach to comparisons of adverse drug reaction reports from patients and healthcare professionals

Collaboration with patient organisations to promote and support patient ADR reporting

Duplicate detection: best practice guidance

Duplicate detection (e-learning)

Feedback to patient ADR reports

Identification, management and raising awareness of ADR reports for drugs subject to additional monitoring

The Strengthening Collaboration for Operating Pharmacovigilance in Europe (SCOPE) WP4—additional monitoring (e-learning)

Medication errors

Tools for measuring and improving the quality of reports in national adverse drug reaction databases

Awareness levels

Adverse drug reactions: reporting makes medicines safer (e-learning for HCPs)

Increasing awareness of national adverse drug reaction reporting systems: best practice guide

Raising and measuring awareness levels for ADR reporting systems through campaigns and regional monitoring centres

Raising awareness of national ADR reporting systems: case studies by country

Strategy guidance for increasing awareness levels of national ADR reporting systems (e-learning)

Reporting tools

Handling telephone calls from the public

The Strengthening Collaboration for Operating Pharmacovigilance in Europe (SCOPE) WP4-telephone reporting (e-learning)

Paper ADR reporting forms

IT systems

IT systems for ADR reporting: best practice guide

Survey reports

SCOPE Work Package 4 Survey Report Topic 1—audit of national reporting systems; 1a Medication errors, 2 Patient reporting, 5 Review of IT systems and special form of reports

Increasing awareness of national adverse drug reaction reporting systems: survey report

SCOPE Work Package 4 Survey Report Topic 4-review of reporting forms

Signal management best practice guide

Introduction to signal management (e-learning)

WP5-literature review on signal management

SCOPE Work Package 5-survey report

Introductory document

Proposals for improvement

Web-based safety information

Risk communication on medicines: report from the workshop (16-17 June 2016)

National strategy for implementation of recommendations on risk communication: key actions

Survey reports and consultations

SCOPE Work Package 6 Survey Report-audit of national methods of communication

SCOPE Work Package 6 Survey Report-web portals

Healthcare Professional Survey-medicines safety communications and their effectiveness

Patient and consumer consultation

\section{Publications}

Communication on safety of medicines in Europe: current practices and general practitioners' awareness and preferences, drug safety

Safety communication tools and healthcare professionals' awareness of specific drug safety issues in Europe-a survey study, drug safety 
Table 2 (continued)

Quality management systems (WP7) Introduction to quality management systems (e-learning)

Compliance and performance: management and indicators

Document and records management

Exchange of information between PV assessors and PV inspectors: best practice guidance

Good practice in the exchange of information between pharmacovigilance assessors and pharmacovigilan ce inspectors within NCAs (e-learning)

Pharmacovigilance quality manual template

Quality planning and quality objectives

Quality management systems: quality planning and quality objectives (e-learning)

Quality standards of pharmacovigilance assessment

Resource management best practice guidance

Resource management best practice (e-learning)

Stakeholder feedback and customer satisfaction

Stakeholder feedback and customer satisfaction: guidance and good practice examples (interactive pdf)

Survey reports

Survey report: understanding national quality systems

Survey report: resource management

Survey report: interaction with pharmacovigilance inspectors

Lifecycle pharmacovigilance (WP8) Competency

Competency recommendations

Available data sources outside spontaneous reports

Identification of available data sources outside spontaneous reports: recommendations

PASS

PASS recommendations

Practical guide on PASS assessment

PASS assessment (e-learning)

PSUR/PSUSA

PSUR/PSUSA and referral recommendations

Practical guide on PSUR/PSUSA assessment

Periodic Safety Update Report (PSUR) assessment (e-learning)

RMPs

Risk management plan recommendations

Practical guide on risk management plan assessment

Risk Management Plan (RMP) Assessment (e-learning)

Safety-related referrals

Practical guide on safety-related referrals

Top 10 tips on safety-related referrals

Safety referrals e-learning: practical guidance for assessors (e-learning)

Survey reports

SCOPE Work Package 8-lifecycle pharmacovigilance executive summary report

The SCOPE website will continue to be live until the end of 2019. Therefore, links in this table may not be accessible from January 2020 but will continue to be available via the EU Network Training Centre and the CHAFEA project database

$A D R$ adverse drug reaction, CHAFEA Consumers, Health, Agriculture and Food Executive Agency, HCPs healthcare professionals, IT information technology, PASS post-authorisation safety and efficacy studies, PSUR periodic safety update reports, PSUSA single assessment of periodic safety update reports, $P V$ pharmacovigilance, $R M P$ risk management plan, $W P$ work package

The web form facilitates submissions of ADRs directly to NCAs. It was designed using the internationally agreed standard for individual case safety reports (ICSRs), and is compatible with the ICH-E2B standard. The system can also act as a database for the management of ADR reports, if required. Following the pilot implementation of the web form in the Romanian regulatory authority ${ }^{1}$ in

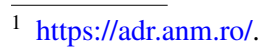


Fig. 2 SCOPE Infographic Reporting suspected sides effects (http://www.scopejoint action.eu/outputsandresults/ adr-collection/awareness-level s/infographic-reporting-suspe cted-side-effects-asset-2/). SCOPE Strengthening Collaboration for Operating Pharmacovigilance in Europe

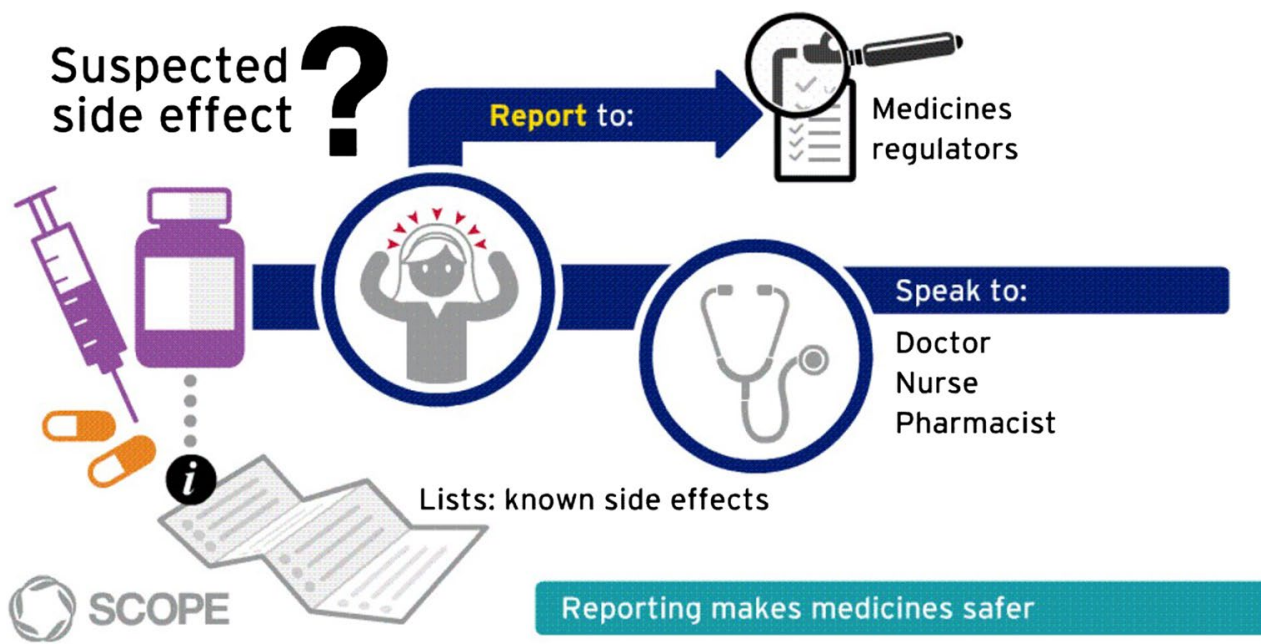

April-October 2016, the tool was made available for implementation in all interested NCAs.

\subsubsection{Awareness Levels Toolkit}

The 2012 legislation also brought in a requirement to encourage the reporting of suspected ADRs [4]. The WP4 survey found that $32 \%$ of NCAs (9/28) did not have a strategy for how to raise awareness levels of national ADR reporting systems. In addition, 68\% (19/28) of the countries did not have a specific budget dedicated to raising awareness. Only four NCAs indicated the use of social media to promote ADR reporting [6]. Therefore, a 'toolkit' was developed with the aim of assisting NCAs in raising awareness of their individual national spontaneous ADR reporting systems. The toolkit ${ }^{2}$ is composed of an animation, three infographics (Fig. 2), an e-learning module providing guidance on a strategy to increase the number and quality of suspected ADRs, and three awareness levels guidance documents (Table 2).

An e-learning module on ADR reporting ${ }^{3}$ was also developed to support HCPs, and this was given an accreditation from the European Accreditation Council for Continuing Medical Education $\left(\mathrm{EACCME}^{\circledR}\right)$ run by the European Union of Medical Specialists (UEMS). Doctors across the EU are awarded 1 EACCME credit for continuing medical education (CME) or continuing professional development (CPD) purposes upon completion of the ADR module which is recognised by National Accreditation Authorities.

\footnotetext{
${ }^{2}$ http://www.scopejointaction.eu/outputsandresults/adr-collection/ awareness-levels/.

3 http://www.scopejointaction.eu/outputsandresults/adr-collection/ awareness-levels/story_html5.html?lms $=1$.
}

\subsubsection{EU-Wide Social Media Campaign to Raise Awareness of National Spontaneous ADR Reporting Systems}

A social media campaign took place as an ADR awareness week on 7-11 November 2016 (Fig. 2). A total of 21 NCAs participated in the campaign, which was the first of its kind in Europe to promote and encourage suspected ADR reporting. The campaign was facilitated through the SCOPE Joint Action working groups and coordinated by the MHRA through the Heads of Medicines Working Group of Communications Professionals (HMA WGCP) [16].

A target of a $5 \%$ increase in the number of suspected ADRs received by NCAs participating in the campaign was set as a goal. The evaluation forms were returned by 15 NCAs, and this showed an overall 13\% increase (1056 reports) in suspected ADR reporting over the campaign, which reached 2,562,071 people. The increase exceeded the target by $8 \%$, which was derived in comparison to ADR reporting baseline measurements provided by Member States [17]. To build on the success of this activity, it was recommended to run similar campaigns in the following years. The HMA WGCP carried out planning and organisation of the second EU-wide ADR awareness week campaign, which took place from 20 to 24 November 2017.

\subsection{Signal Management (WP5)}

\subsubsection{Best Practice Guide on Signal Management and e-Learning}

Using WP5 survey data and other available data sources, a best practice guide was developed for the European 


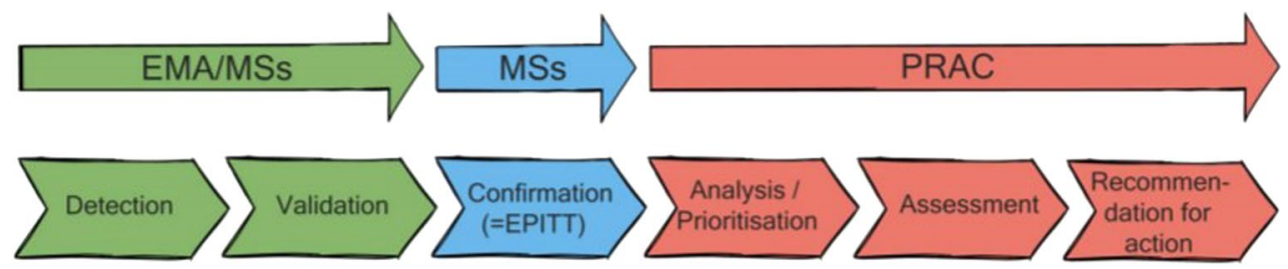

Fig. 3 Signal management processes/responsibilities and activities for regulators, as provided in the legislation [18]. EMA European Medicines Agency, MSs member states, EPITT European Pharmacovigilance Issues Tracking Tool, PRAC Pharmacovigilance Risk Assessment Committee

network. ${ }^{4}$ The best practice guide provides recommendations for efficient and effective signal management at Member State level, regardless of the size, NCA structures or resources, and clearly outlines the various steps involved in signal management: detection, validation, confirmation, analysis/prioritisation, assessment, recommendation for action (Fig. 3). The recommendations from this guide, together with the legal requirements on signal management and GVP guidance, aim to facilitate current signal management in the EU [18].

In addition to practical recommendations, the best practice guide also provides recommendations for the EMA and the EU Regulatory Network to consider for future research and development, aimed at improving signal management within the EU regulatory network. This covers both processes and IT tools used within signal management and demonstrates the possibility to improve efficiency within the system. The WP5 recommendations refer to general areas in signal management, such as access to data, exchange of information and tracking of signals, and more specific steps in the signal management process, as well as reports of special interest.

The WP5 results indicate that the existing heterogeneity in the process of signal detection methods within the EU network appears to be beneficial for the whole system. The differences in the national databases and the different methods applied can be considered a strength of the system. Especially with regard to the signal detection process, it is important that the methods applied are appropriate for the respective databases and there is no 'one size fits all' solution. Therefore, the diversity in databases and methodologies allows the detection of different signals in the different databases [18].

The best practice guide has been complemented with an e-learning module ${ }^{5}$ that serves as an introduction to the topic, together with training sessions covering signal

\footnotetext{
${ }^{4} \mathrm{http}: / / \mathrm{www}$. scopejointaction.eu/outputsandresults/signal-manag ement/.

5 http://www.scopejointaction.eu/outputsandresults/signal-manag ement/story_html5.html?lms=1.
}

management, with a primary focus on the process at the Member State level [18].

An extensive literature review on the topic of signal management was also performed. A relatively large amount of research available regarding signal management was found, most of which focused on signal detection, including interesting and relevant research within the Pharmacoepidemiological Research on Outcomes of Therapeutics by a European Consortium (IMI PROTECT) project. Other topics such as signal validation, prioritisation and assessment are less well-explored scientifically and further scientific work in these areas is recommended in order to improve the quality and consistency of decision making in the context of managing signals.

\subsection{Risk Communication (WP6)}

\subsubsection{Medicines Safety Communications and Their Effectiveness: Awareness and Preferences of Healthcare Professionals}

WP6 performed a study between June and September 2015, aimed at assessing awareness and preferences of HCPs for risk communication. This study highlighted the familiarity of European HCPs with the main safety communication tools utilised by NCAs and industry. General practitioners (GPs), pharmacists and cardiologists were the target population for this survey, which was distributed among HCPs in nine European countries (Denmark, Spain, Croatia, Ireland, Italy, The Netherlands, Norway, Sweden, and the UK). A 5 -point Likert scale ranging from very negative (1) to very positive (5) was used to measure preferences for senders and channels. The questions were also customised for each country to provide local information to responders, if necessary [9].

WP6 found that the most trusted senders of safety information were the NCAs and professional bodies, while the least-valued distributors were considered lay press and pharmaceutical companies. GPs' awareness of Direct Healthcare Professional Communications (DHPCs) and NCA communications was high, i.e. 91 and 79\%, respectively. However, awareness of the educational materials among GPs was 
lower at $64 \%$. The preference for an electronic format rather than a hard copy version varied among countries. Of the respondents, $89 \%$ considered repetition of messages as useful. GPs recognised point-of-care alerts and email as the most preferred alternative communication channels [9].

Familiarity with DHPCs was also high among cardiologists and pharmacists across Europe. In general, cardiologists were more aware than GPs of the safety issue for a drug within their field of expertise, and less aware than GPs and pharmacists on safety issues regarding other drugs, even though some of these related to cardiovascular risks. The DHPCs were an important source of safety information for GPs, cardiologists, and pharmacists to become aware of specific safety issues, followed by other sources of information, such as websites or newsletters and medical journals. The study implies that NCAs may need to use additional safety communication strategies to reach specialists when informing about risks associated with medicines from outside their areas of expertise, but which have safety risks relating to their specialisation [19].

The information from this study was used alongside data from two other WP6 surveys aimed at NCAs, to form part of the proposals for improving the risk communication overview developed by WP6.

\subsubsection{Patient and Consumer Consultation}

WP6 consulted with European patients and consumer organisations, and their views were gathered with the help of an 'aid-memoire', asking about educational materials, safety review communications/transparency, awareness of the regulatory system, and familiarity with side-effect reporting tools. The consultation was answered by 11 European associations representing seven countries (Belgium, Spain, Portugal, Ireland, Italy, Lithuania and Macedonia). Respondents highlighted that educational materials could function as tools to encourage discussion of safety information between patients and HCPs; HCPs were considered the most trusted source of information, and publication of educational materials on NCA websites could also improve transparency. The consultation report also implies the importance of measures to enhance awareness of the regulatory system and how it works among patient and consumers, and that the development of links with patient and consumer organisations could help to raise awareness of the NCA role [20]. In addition, information regarding the ADR reporting tools was shared with WP4.

\subsubsection{Web Portals Good Practice Guide and Summary of Proposals for Improvements}

The WP6 surveys gained insight into the current systems, mechanisms and practices used by European NCAs to communicate safety messages $[10,11]$. In addition to these surveys, NCAs reviewed each other's websites and provided comments on the methods for communicating safety information. From these reviews and survey responses, case studies for good practice were drawn out and developed into presentations in the good practice guide-Web-Based Safety Information. The guide provides case studies of how NCAs present their national pharmacovigilance information, giving both examples of good practice and examples where NCAs have gone 'above and beyond' in their methods. Furthermore, it supports NCAs to meet the requirements set out in the EU pharmacovigilance legislation.

WP6 summarised all the recommendations from all three WP6 surveys and consultations, and prepared a summary of proposals for improvement of risk communications. ${ }^{6}$ This document is intended to provide practical guidance on selected aspects of risk communication, and a useful overview that could be adapted to local/national systems (Table 2).

\subsubsection{Workshop on Risk Communication on Medicines}

In 2016, WP6 organised a workshop on risk communication on medicines to disseminate results and outputs from WP6 studies, to learn from each other's experience, and to discuss how EU national agencies can improve their communication on the risks associated with medicines. Participants representing patients and consumer organisations; HCPs; academia; pharmacovigilance assessors and communications experts from European NCAs; and other stakeholders representing European, Global and Ibero-American organisations working on pharmacovigilance topics, attended the workshop. Breakout sessions provided an opportunity to discuss the following aspects: public participation in risk communication activities, communication of emerging safety issues, dissemination of messages to target audiences, and use of supplementary materials as additional risk minimisation tools. A report summarising panel discussions and ideas raised by participants was collated and published on the SCOPE website. ${ }^{7}$

The risk communication workshop reflected on how risk communication practice could move forward in the near future. It was unique in that participants with different profiles were able to express their opinions and awareness. Furthermore, some participants suggested that there is a need for a European forum involving NCAs and academia representatives to develop a consistent strategy for risk communication. In conclusion, the attendees indicated the importance

\footnotetext{
$\overline{6}$ http://www.scopejointaction.eu/_assets/files/WP6-Risk-Commu nication-Proposals-for-Improvement.pdf.

${ }^{7}$ http://www.scopejointaction.eu/events/wp6-workshop/.
} 
of exchanging experiences between the regulators and citizens and how this rewarding experience could benefit the communication process. A report on the workshop ${ }^{8}$ and a document entitled National Strategy for Implementation of Recommendations on Risk Communication: Key Actions ${ }^{9}$ were prepared.

In the post-work evaluation activities, attendees emphasised interest in the topic of risk communication and a willingness to continue a multidisciplinary collaboration to improve and strengthen risk communication activities. Following on from the workshop, a guide for NCAs in the process of implementing recommendations from SCOPE WP6 was developed. As the level of complexity, and the resources needed, differ for the various key actions suggested, this guide gives some tips to help the Member States during the implementation process.

\subsection{Quality Management Systems (WP7)}

\subsubsection{Quality Management Systems Site Visits}

In May and June 2014, WP7 organised five site visits to NCAs in The Netherlands, Lithuania, Spain, Czech Republic and Ireland. Discussions during the site visits focused on identifying examples of best practice in the quality management systems in pharmacovigilance and gaining a better understanding of how Member States have been interpreting the pharmacovigilance legislation and guidance available. Results from the site visits informed development of the WP7 survey on quality management systems.

\subsubsection{A Toolkit That Can Be Used for Further Development of Quality Management Systems}

WP7 created a toolkit ${ }^{10}$ that can be used to further develop NCAs' quality management systems in the area of pharmacovigilance business activities. The toolkit is a diverse collection of case studies, good practices, templates and guidance for NCAs to aid the understanding of quality management and the establishment and operation of their pharmacovigilance quality systems. Tools developed by WP7 aim to increase existing knowledge on quality management systems enhancing the quality of pharmacovigilance services provided by concerned stakeholders, to provide guidance on exchange of information between pharmacovigilance

\footnotetext{
8 http://www.scopejointaction.eu/_assets/files/WP6-Risk-Commu nication-on-Medicines-Workshop-Report.pdf.

9 http://www.scopejointaction.eu/_assets/files/WP6-Key-Actions-forImplementation-of-Risk-Communication.pdf.

$10 \mathrm{http} / /$ www.scopejointaction.eu/outputsandresults/quality-manag ement-systems/.
}

assessors and inspectors, quality planning and quality objectives, quality standards for pharmacovigilance assessment, resource management, compliance and performance management, gathering stakeholder feedback and measuring customer satisfaction, with a special focus on setting up or improving pharmacovigilance quality manuals, and document management systems (Table 2).

\subsection{Lifecycle Pharmacovigilance (WP8)}

\subsubsection{Strengthening Capabilities for Benefit- Risk Assessment: Practical Guidance for Pharmacovigilance Assessors}

Improvement and harmonisation of benefit-risk assessment, and its implementation throughout the EU, was emphasised in the pharmacovigilance legislation [Regulation (EU) No 1235/2010 and Directive 2010/84/EU]. Development of a unified approach to benefit-risk assessment, use of available tools, and strengthening of NCAs' competencies to effectively assess benefit-risk of medicines were recognised as essential to strengthen NCAs' capabilities and to support the safety of patients, and public health. WP8 was set up to promote the importance of consistency, and a systematic approach in pharmacovigilance assessment through the lifecycle. During the project, information on existing methods and processes for pharmacovigilance procedures was collected, and descriptive survey reports were delivered.

Fourteen outputs ${ }^{11}$ aiming to support pharmacovigilance practice throughout the product lifecycle were created by WP8 (Table 2). These deliverables are intended to provide recommendations, practical guidance and training on some specific areas of pharmacovigilance assessment, in addition to formal regulatory guidelines and national standard operating procedures (SOPs). Four e-learning modules were designed to maximise training opportunities for pharmacovigilance assessors (Table 2). WP8 outputs were based on the survey data analysis and practical experience in pharmacovigilance assessment procedures. These tools aim to provide practical guidance on handling pharmacovigilance assessment procedures, and on key challenges and learning identified via the survey. WP8 outputs were written and created by experienced pharmacovigilance assessors to provide practical advice on some aspects of pharmacovigilance assessment to assessors in Europe.

\footnotetext{
$\overline{11} \mathrm{http} / / / \mathrm{www}$. scopejointaction.eu/outputsandresults/lifecycle-pharm acovigilance/
} 


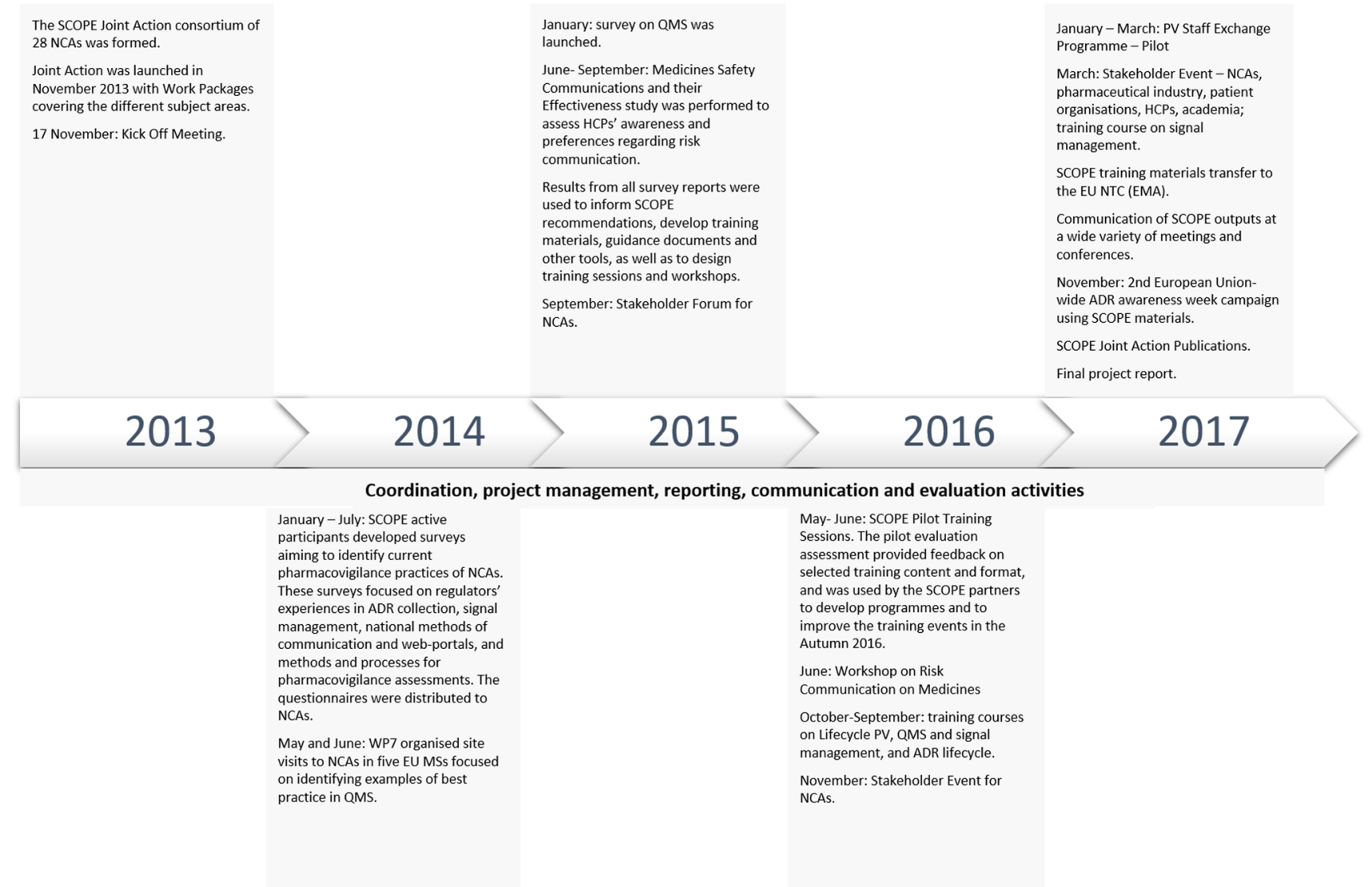

Fig. 4 Key project activities. SCOPE Strengthening Collaboration for Operating Pharmacovigilance in Europe, NCAs national competent authorities, $A D R$ adverse drug reaction, EMA European Medicines

\subsubsection{Pharmacovigilance Staff Exchange Programme: Pilot}

The continued benefit-risk assessment of a medicinal product throughout its lifecycle in clinical use is essential for the effective operation of the pharmacovigilance system in the EU. Improvement of assessor skills in this field, and harmonisation of the assessment process among European Member States, are two of the most important aspects of the new pharmacovigilance legislation and its implementation throughout the EU. Relying on the competence of other NCAs also reduces duplication of effort, shares the workload, and ensures the efficient and effective regulation of medicines across the EU [21].

WP8 survey results strongly indicated that a programme supporting the exchange of experience and knowledge among assessors was considered a valuable tool to strengthen pharmacovigilance assessment activities. The WP8 questionnaire on RMPs found that on-the-job training, such as learning from reading others' assessment reports (92\%) and mentoring by senior assessors $(75 \%)$ were the most common forms of training in NCAs. When questioned on any other challenges regarding assessment of RMPs,
Agency, EU NTC European Union Network Training Centre, $H C P$ healthcare professionals, $M S s$ member states, $P V$ pharmacovigilance, $Q M S$ quality management systems, $W P$ work package

sharing information and knowledge with more experienced colleagues from other NCAs was explicitly mentioned [15, 22].

A pilot exchange programme was established as a proof of concept to investigate feasibility and to propose grounds for a sustainable programme for interactions among European NCAs. This programme aimed to provide on-the-job training, promote close collaboration, and harmonise standards for pharmacovigilance procedures. Finally, it would strengthen effectiveness of the network and support the work of the PRAC.

In 2017, three participants from two NCAs (Spain, Norway) who expressed interest in learning more on pharmacovigilance assessment and associated activities took part in the pilot exchange programme. The duration of individual training programmes was between two and four weeks duration in the period between January and March 2017, and depended on the training aims and content. Three NCAs responsible for the regulation of human medicines in the EEA offered to host, and provided training (Spain, Norway, and Portugal). Evaluation reports received from both the hosting organisations and participants provided evidence 
that the programme could be an effective way of training and sharing knowledge across the pharmacovigilance network.

\section{Bringing People Together}

\subsection{SCOPE Joint Action Training Workshops}

In September and October 2016, training courses were held in Lisbon, Budapest, and London; these events focused on the areas of lifecycle pharmacovigilance, quality management systems and signal management, and ADR lifecycle, respectively (Fig. 4). A second training session on signal management reflecting findings from SCOPE WP5 was organised in April 2017 in Utrecht, The Netherlands. Participants from the majority of European NCAs attended SCOPE training sessions. These training sessions were preceded by two pilot training events organised in May and June 2016. The pilot evaluation assessment provided feedback on selected training content and format and was used by the SCOPE partners to develop programmes and improve the main training events.

In March 2017, a joint training session with the European Organisation for Rare Diseases (EURORDIS) was organised in London. This event brought together 30 patient organisation representatives and was organised to support patient engagement, support learning about pharmacovigilance, and to receive training in responding to challenging telephone calls in relation to suspected ADRs.

\subsection{Key Stakeholder Events}

In 2015 and 2016, two stakeholder events on SCOPE findings and outputs for NCAs were held in London. A wider stakeholder event was held in March 2017 in London, and the event brought together key stakeholders from NCAs, pharmaceutical industry, patient organisations, HCPs, and academia (Fig. 4).

The SCOPE stakeholder events provided an opportunity to raise awareness of the research and work carried out by the Joint Action across EU NCAs and other stakeholder groups, and to discuss and explore the project's findings. Suggestions arising from the stakeholder event in March 2017, in relation to recommendations for the future, included continuing to build on the work produced through the SCOPE network, the need for future collaboration activities to strengthen the pharmacovigilance system, and bringing together the key players to work to further develop and take forward ideas. More engagement with patient organisations and a suggestion to further consider a concept of having a 'qualified' contact person leading on pharmacovigilance activities in patient organisations were also raised. There was also a keenness from the industry for some face-to-face training to be made available to them.

The SCOPE partners also organised local stakeholder engagement meetings with patient organisations in Hungary and Croatia (March and April 2017). These meetings provided an excellent opportunity for participants to familiarise themselves with the procedures and benefits of reporting ADRs, and emphasised the need for their contribution to the medicines safety. At the local stakeholder event held in Budapest (March 2017), further to the oral presentations of the SCOPE project findings, printed SCOPE materials conveying the main messages of the project were prepared and distributed among the targeted stakeholders (patients, patient organisations, and HCPs) in Hungary with great success.

In Croatia, the 'Monitoring Medicines Safety' event gathered around 50 representatives of patient associations and life sciences students. The value and findings of SCOPE, as well as the significance of the role of patients and HCPs in monitoring the safety of medicines, were presented and discussed through a series of lectures, discussions, a workshop and a round table meeting.

\section{Value of the SCOPE Joint Action: Who Else Can Gain?}

In the context of NCAs' diversity and variety of business practices, strengthening of assessors' competency and continuous professional development is important to ensure effectiveness of the whole pharmacovigilance network and European medicines regulatory system. Relying on the competence of other NCAs also reduces duplication of effort, shares the workload, and ensures the efficient and effective regulation of medicines across the EU.

The target audience of the SCOPE outputs is primarily pharmacovigilance staff working at European NCAs. However, SCOPE outputs can be useful for other NCA staff, such as policymakers from national agencies or regional pharmacovigilance centres. It may also be helpful for other stakeholders involved in pharmacovigilance activities, such as Marketing Authorisation Holders (MAHs), HCPs and patient organisations. The SCOPE Joint Action developed several approaches to enhance sustainability, and to maximise the benefits of its outputs, including collaboration with both the EU NTC and the Pharmacovigilance Training Curriculum (PhVTC), and use of the SCOPE website. ${ }^{12}$

The EU NTC has created a platform for the exchange of information and supply of regulatory and scientific training across the EU regulatory network. The intention of SCOPE was that the project outputs will stimulate and

$\overline{12}$ http://www.scopejointaction.eu/. 
provide resources for other ongoing initiatives within the network. Therefore, a number of training materials that were developed through the SCOPE Joint Action have been made available to the network via the EU NTC platform. These resources may be particularly helpful for new members of pharmacovigilance staff. In addition, the International Society of Pharmacovigilance (ISoP) was also keen to collaborate and share SCOPE training materials more widely through the ISoP pharmacovigilance training platform. The SCOPE Joint Action outputs have been highlighted at a wide variety of meetings and conferences, both within the EU and at a global level, e.g. WHO Collaborating Centres and Drug Information Association, and there has been interest from a range of different stakeholders in using the materials.

The SCOPE Joint Action results strongly indicated that an exchange programme for EU pharmacovigilance staff is considered a valuable tool to share experience and knowledge among NCAs. This initiative provides on-the-job training, promotes close collaboration, and harmonises standards for pharmacovigilance procedures. It is expected that, in the long term, it would improve effectiveness of the network, especially the work of the PRAC. The SCOPE pilot programme was set up as a proof-of-concept to investigate feasibility and establish grounds for a sustainable programme for interactions among European NCAs. Evaluation reports received from both the hosting organisations and participants provided evidence that the programme could be an effective way of training and sharing knowledge across the pharmacovigilance network.

The SCOPE Joint Action also focused on increasing awareness levels of national spontaneous ADR reporting systems. A 45-min e-learning package about ADR reporting was developed to support HCPs. This module received accreditation from the $\mathrm{EACCME}^{\circledR}$, and doctors across the EU are awarded 1 EACCME credit upon completion of the ADR module which is recognised by National Accreditation Authorities.

The ADR media materials intended for promoting the reporting of suspected ADRs can continue to be used by the NCAs and wider stakeholders. Tailored versions of infographics were produced in different EU languages, and these materials can be used in coordinated EU campaigns or by individual NCAs. Different key messages can be given, e.g. focusing on children or over-the-counter products, etc., depending on the focus of a campaign.

The Joint Action gave the opportunity for pharmacovigilance staff across the network to meet each other during face-to-face meetings and training events, enabling them to more confidently contact and liaise with their peers in other countries. This has been invaluable for helping to build the informal network and develop contacts for knowledge and resource-sharing purposes.

\section{Discussion}

The SCOPE Joint Action was the first initiative of its kind to be established in Europe to support the operation of pharmacovigilance systems and to strengthen the regulatory network. A number of new aspects were introduced in the pharmacovigilance legislation, which came into effect in July 2012 [Regulation (EU) No 1235/2010, Directive 2010/84/EU]. First, patients are well placed to report suspected adverse reactions to medicinal products, and the NCAs should facilitate patient reporting through provision of various reporting formats. The NCAs have an overall responsibility to improve and encourage reporting of suspected ADRs within their respective countries, including collection of information about off-label use. The legislation introduced an EU-wide process for signal management overseen by the PRAC, with specific responsibilities and interactions among all stakeholders involved. The PRAC came into existence in July 2012 to strengthen the oversight of safety monitoring of medicines across Europe; its mandate covers all aspects of risk management of medicinal products for human use. The legislation included a number of provisions to strengthen safety communication and its coordination, and stated that Member States have to create and maintain a web portal (website) for making information on medicines publicly available in order to increase the level of transparency of pharmacovigilance processes and outcomes. The legislation also stipulates legal requirements for quality management systems, and the continued benefit-risk assessment of a medicinal product lifecycle. This was considered a cornerstone for the effective operation of the pharmacovigilance system in the EU.

The pharmacovigilance legislation is supported by the GVP modules, a set of guidelines developed to facilitate the performance of pharmacovigilance in the EU, including centralised and national procedures. GVPs apply to medicines regulatory authorities in EU Member States, MAHs, and the EMA. The SCOPE outputs were created to complement the GVP modules, and concentrated on the practical aspects of collecting information on ADRs, signal management, communicating risk and assessing risk minimisation measures, supported by effective quality management systems. The SCOPE project developed approximately 70 deliverables, including guidance documents with case studies, e-learning modules, other learning materials, survey reports, publications, and other tools. Face-to-face training events for NCAs and stakeholder events were also held.

The SCOPE outputs have also helped to inform updates to GVP modules, for example the key WP5 deliverable, the Best Practice Guide on Signal Management, was taken into account during the revision of the GVP IX Revision 1 (22 November 2017) [23]. 
In addition to the implementation of this major regulatory revision, some European regulatory agencies have been actively engaging in initiatives and projects, with the aim of improving the quality of pharmacovigilance systems and seeking to utilise and explore the power of new technologies for pharmacovigilance purposes. Two Innovative Medicines Initiatives (IMI) projects-the Pharmacoepidemiological Research on Outcomes of Therapeutics by a European ConsorTium (PROTECT), and the WEB-RADR: Recognising Adverse Drug Reactions (WEB-RADR) - overlapped to some extent with the SCOPE Joint Action. Like PROTECT, SCOPE has researched the topic of signal detection. The remit and focus of these two initiatives were very different from SCOPE, however each initiative was aware of progress and any areas of overlap.

Unlike the IMI PROTECT initiative, which aimed to develop new methods and assess existing ones for signal detection from spontaneous reports, electronic health records and clinical trials [24], SCOPE focused on the regulatory processes in the Member States and provided recommendations and guidance for efficient and effective signal management at Member State level. Most of the SCOPE recommendations were based on the outputs of the WP5 survey conducted among Member States, where best practices were identified [8].

Provision of new ADR reporting tools was investigated by the IMI WEB-RADR project. This project developed and deployed user-friendly mobile applications (apps) in three countries (UK, The Netherlands, Croatia) and, subsequently through WHO interest, in Burkina Faso and Zambia. The apps facilitate direct and instant reporting of suspected ADRs by patients, HCPs and the public, and the two-way communication of up-to-date pharmacovigilance information [25]. In contrast, SCOPE focused on strengthening systems for web-based and traditional reporting practice. A web form for various reporter groups was created, including a content management system that can act as a database for NCAs who do not have a system in place. There was close collaboration between both projects, and WEB-RADR used infographics developed by SCOPE to raise awareness of national ADR reporting systems during the mobile app launches in Africa.

The IMI WEB-RADR and PROTECT initiatives were collaborations with pharmaceutical companies and other bodies, while SCOPE Joint Action was a unique initiative designed and delivered by NCAs for NCAs. The SCOPE project established links with these projects, elaborated on some results from PROTECT and shared knowledge with WEB-RADR, to maximise the use of funding and avoid duplication of efforts. The SCOPE's research focused on the NCAs' work and strengthening pharmacovigilance practice across Europe. Therefore, the majority of SCOPE funding was budgeted to cover work delivered by the NCA staff.
Collaboration of European medicines regulators made it possible to achieve the project's goals, with valuable input from other stakeholders, by developing scientific outputs and providing responses to SCOPE surveys.

The SCOPE initiative differs from all the other projects as it was the first initiative dedicated entirely to strengthening national pharmacovigilance activities, and it was created by and for NCAs. The SCOPE achievements are a response from national medicines regulators to the implementation of the pharmacovigilance legislation contained in Regulation (EU) No 1235/2010 and Directive 2010/84/EU. The intention for the SCOPE outputs was that NCAs can use these tools (guidance and training materials) to meet their needs and take from them any ideas that they consider useful to strengthen or develop their regulatory systems and business practices. However, it was identified during the stakeholder meetings that SCOPE outputs may also be helpful to other stakeholders involved in pharmacovigilance activities, such as the pharmaceutical industry, patients and HCPs. Medicines regulators from outside Europe could also benefit from SCOPE tools, especially those interested in development of their pharmacovigilance system, as the tools present a variety of business practices.

Pharmacovigilance and pharmacoepidemiology courses are offered by different organisations in Europe, e.g. the European programme in Pharmacovigilance and Pharmacoepidemiology (EU2P). However, SCOPE was set up to deliver face-to-face training courses and learning materials for NCAs focusing on specific aspects identified via the SCOPE surveys. These training events also provided an opportunity to disseminate information about SCOPE research and to build and strengthen collaboration among NCAs. A key benefit of the SCOPE Joint Action was that it provided a unique opportunity to bring together colleagues from across the NCA network and discuss issues, and also to increase closer working relationships.

The SCOPE Joint Action demonstrated how national medicines regulators can work together to strengthen and develop capabilities within the network. National medicines regulators recognised the significance of SCOPE research and provided a large amount of information about their national pharmacovigilance systems and strategies. Depending on the survey, between 25 and 28 NCAs returned the questionnaires, which included a high number of individual free-text comments to open-ended questions. This indicates a real interest in the area and a high ambition for improvement among NCAs, even though survey fatigue was indicated by some NCA respondents who became overwhelmed with questions and the number of surveys they were asked to complete.

The prioritisation of resources available in the pharmacovigilance network to deliver the work, respond to surveys, and participate in training events was a challenge for the 
Joint Action and the pharmacovigilance network, particularly NCAs with less resources available. Other challenges related to tight timeframes, high amount of outputs planned to be delivered in the final stage of the project, along with organisation of training events and workshops. In addition, appropriate distribution of funds to develop training materials for all of the different WPs was challenging.

There was a specific WP responsible for the measurement and assessment of SCOPE activities, and regular reports were given to the WP Leaders Group and the General Advisory Board. Evaluation throughout the Joint Action helped to ensure that the project remained on track and that the deliverables were being achieved in line with the Grant Agreement. It provided a tool for improvement and was used when further developing the training from pilot to the final training sessions. Specific processes, outputs and outcome indicators were developed in the initial stage of the Joint Action, and SCOPE results were measured against these. The Joint Action achieved all of its objectives and carried out additional activities, such as the social media awareness campaign and the pilot exchange programme for pharmacovigilance staff.

In summary, the network showed extremely effective collaboration, helping each other, sharing knowledge and avoiding duplication of effort. The work was coordinated with clear roles and responsibilities, with each WP having the autonomy to take forward the different strands of work, but coming together to ensure consistent, consolidated products and training. The input, guidance and steering from members of the General Advisory Board were invaluable, giving a broad overview and direction, managing risks and building the links to wider stakeholders.

Part of the success of the SCOPE Joint Action was through using the practical experience and skills already available in the network, while another factor was the keenness of those involved to make best use of the opportunity and collectively make a difference to pharmacovigilance operations across Europe. The SCOPE Joint Action has been an opportunity to highlight the NCAs' current practice (via surveys, training events, stakeholder engagement activities), and to identify how to move forward and improve activities in the network. Heterogeneity of systems and methods was reflected in SCOPE results and outputs and can be considered to be a strength of the system. Consequently, examples of various methods and business practices were included in guidance documents, recommendations, and e-learning modules.

In terms of future use of SCOPE research, the HMA initiative on Better Use of Medicines will contribute to the sustainability of the WP6 outputs and results. Initially, the group will investigate how the SCOPE WP6 outputs have been used by European NCAs. This Working Group was established to coordinate activities related to improving communication tools for patients and HCPs aiming to enhance the use of medicines, including embracing new approaches to optimise communication in different media, tailoring guidance on prescriptions and improving information to patients.

Although SCOPE was considered a one-off initiative, European projects that are targeted to NCAs and devoted to the improvement of pharmacovigilance activities could be considered in the future, given the success of SCOPE and its achievements. In addition, other multicountry initiatives and projects may find SCOPE a useful model for collaborative working. The project management and governance structure could be successfully replicated for other Joint Actions or similar projects. The SCOPE outputs were shared with the network via training meetings and workshops, and transferred to the EU NTC for inclusion in the pharmacovigilance curriculum with the view of the materials being kept under review and updated when appropriate.

\section{Conclusions}

The SCOPE Joint Action was established in Europe to support operation of pharmacovigilance systems and to strengthen the national regulatory network, and was created by and for NCAs. The SCOPE project brought together national medicines regulators from the EEA to develop guidance, training in key aspects of pharmacovigilance, and tools and templates to support best practice for NCAs. Medicines regulators have worked together to improve the skills and capability in the pharmacovigilance network to help safeguard public health in both national territories and the EU as a whole. The SCOPE training materials and outputs have been made publicly available for interested parties to use and implement according to their needs.

Acknowledgements The views expressed in this paper are those of the authors only and not of their respective institution.

The authors would like to thank the EU and the CHAFEA for supporting this Joint Action; the support of the EU was essential to successfully achieve all the Joint Action goals. The success of the Joint Action would not be possible without the assistance of the SCOPE General Advisory Board, who provided strategic advice throughout the project, therefore enabling the delivery of high standard outputs and results. SCOPE Joint Action General Advisory Board Members and representatives from the EC and CHAFEA included (in alphabetic order): Luis Correia Pinheiro, François Houÿez, Helen Lee, Hervé Le Louet, Marie Lindquist, Georgios Margetidis and June Raine. The authors would also like to thank Franck Diafouka, a former General Advisory Board member, for his contribution to this project. The authors gratefully acknowledge the SCOPE Joint Action Topic Leaders and Active Partners for their tremendous contribution to the project. Representatives from the following National Competent Agencies made invaluable contributions to the work of the SCOPE work packages (in alphabetic order): Agencia Española de Medicamentos y Productos Sanitarios (Spain), Bulgarian Drug Agency (Bulgaria), Danish Health Authority (Denmark), Fundación Española para la Cooperación 
Internacional, Salud y Política Social (Spain), HALMED Agency for Medicinal Products and Medical Devices (Croatia), Health Products Regulatory Agency (Ireland), INFARMED National Authority of Medicines and Health Products (Portugal), Italian Medicines Agency (Italy), Medicines \& Healthcare products Regulatory Agency (UK), Medicines Evaluation Board (Netherlands), National Agency for Medicines and Medical Devices (Romania), National Institute of Nutrition (Hungary), Norwegian Medicines Agency (Norway), State Institute for Drug Control (Czech Republic), State Medicines Control Agency (Lithuania) and Swedish Medical Products Agency (Sweden). The collaboration of all partners made it possible to achieve the project's goals, by developing scientific outputs and providing responses to SCOPE surveys and participating in the training and stakeholder engagement meetings. The authors are grateful for the support provided by all project partners, and invaluable contribution to the Joint Action. Other partners included (in alphabetic order): Agence Nationale de Sécurité des Médicaments et des Produits de Santé (France), Agency for Medicinal Products and Medical Devices of the Republic of Slovenia (Slovenia), Federal Agency for Medicines and Health Products (Belgium), Finish Medicines Agency Fimea (Finland), Icelandic Medicines Agency (Iceland), Medicines Authority (Malta), National Organisation for Medicines (Greece), Office for Registration of Medicinal Products, Medical Devices and Biocidal Products (Poland), Pharmaceutical Services of Ministry of Health (Cyprus), State Agency of Medicines (Estonia), State Agency of Medicines of Latvia (Latvia) and State Institute for Drug Control (Slovakia). The authors are also grateful for the contribution from a number of other organisations with specific interests and expertise in pharmacovigilance topics (in alphabetic order): Maastricht University, Netherland Pharmacovigilance Centre Lareb, University Medical Centre Groningen, University of Nottingham and Uppsala Monitoring Centre.

\section{Compliance with Ethical Standards}

Ethical approval was not considered necessary for this work.

Sources of funding The SCOPE Joint Action was funded by the Health Programme of the European Union 2008-2013, with contribution from the involved member states, under Grant Agreement No. 20132102. Full details of the awarding agency, the CHAFEA, can be found at http://ec.europa.eu/chafea/index.html. The open access fee for this publication was paid by the grant received from CHAFEA.

Conflict of interest Anna Radecka, Louise Loughlin, Mick Foy, Margarida Guimaraes, Viola Macolic Sarinic, Marina Dimov Di Giusti, Marina Lesicar, Sabine Straus, Dolores Montero, Julia Pallos, Jelena Ivanovic, and June Raine have no conflicts of interest that are directly relevant to the content of this article.

Open Access This article is distributed under the terms of the Creative Commons Attribution-NonCommercial 4.0 International License (http://creativecommons.org/licenses/by-nc/4.0/), which permits any noncommercial use, distribution, and reproduction in any medium, provided you give appropriate credit to the original author(s) and the source, provide a link to the Creative Commons license, and indicate if changes were made.

\section{References}

1. European Commission. MEMO/08/782, Brussels, 10 December 2008. Strengthening pharmacovigilance to reduce adverse effects of medicine. Available at: http://europa.eu/rapid/press-release_ MEMO-08-782 en.htm?locale=en. Accessed 1 Feb 2018.

2. Pirmohamed M, James S, Meakin S, Green C, Scott AK, Walley TJ, et al. Adverse drug reactions as cause of admission to hospital: prospective analysis of 18,820 patients. BMJ. 2004;329:15-9.

3. Santoro A, Genov G, Spooner A, Raine J, Arlett P. Promoting and protecting public health: how the European union pharmacovigilance system works. Drug Saf. 2017;40(10):855-69.

4. Directive 2010/84/EU of the European Parliament and of the Council of 15 December 2010. Available at: http://eur-lex.europ a.eu/LexUriServ/LexUriServ.do?uri=OJ:L:2010:348:0074:0099 :EN:PDF. Accessed 22 Nov 2017.

5. Šarinić VM, Dimov Di Giusti M, Banovac M, Skvrce NM, Gvozdanović K, Krnić D, et al. SCOPE Work Package 4 survey report. Available at: http://www.scopejointaction.eu/_assets/files /SCOPE-WP4-Topic-1,2,5-survey-report.pdf. Accessed 22 Nov 2017.

6. Jadeja M, Barrow P. SCOPE Work Package 4. ADR collection: increasing awareness of national ADR reporting systems: survey report. Available at: http://www.scopejointaction.eu/_assets/files/ WP4.3-Survey-Report-layout-draft-4-final.pdf. Accessed 22 Nov 2017.

7. Jan T, Radecka A. SCOPE Work Package 4 survey report. Topic 4 review of reporting forms. Available at: http://www.scopejoint action.eu/_assets/files/SCOPE-WP4-Topic-4-Survey-Report-v0-7.pdf. Accessed 22 Nov 2017.

8. Pacurariu A, van Haren A, Berggren AL, Grundmark B, Vestergaard Laursen M, et al. SCOPE WP5 survey report. Available at: http://www.scopejointaction.eu/_assets/files/SCOPE-WP5-FULLreport.pdf. Accessed 22 Nov 2017.

9. De Vires ST, van der Sar MJM, Cupelli A, Baldelli I, Coleman $\mathrm{AM}$, Montero D, et al. Communication on safety of medicines in Europe: current practices and general practitioners' awareness and preferences. Drug Saf. 2017;40(8):729-42.

10. Rastad JA, Forrest M, Wennberg A. SCOPE Work Package 6 survey report audit of national methods of communication. Available at: http://www.scopejointaction.eu/_assets/files/SCOPE-WP6-Topic-1-FINAL-report.pdf. Accessed 22 Nov 2017.

11. Haddad R, Barrow P. SCOPE Work Package 6 survey report webportals. Available at: http://www.scopejointaction.eu/_assets/files /SCOPE-WP6-Topic-4-Final-survey-report-v-0-2.pdf. Accessed 22 Nov 2017.

12. Pálfi M, Cserjés Z. SCOPE Work Package 7 quality management systems survey report: understanding national quality systems. 2015. Available at: http://www.scopejointaction.eu/_assets/files /Quality-Survey-Report.pdf. Accessed 22 Nov 2017.

13. Chadda K, Barrow P, Cserjés Z, Hearn J. SCOPE Work Package 7 quality management systems survey report: resource management. 2016. Available at: http://www.scopejointaction.eu/_assets/ files/Resource-Management-Survey-Report.pdf. Accessed 22 Nov 2017.

14. Chadda K, Barrow P, Webb R, Cserjés Z, Hearn J. SCOPE Work Package 7 quality management systems survey report: interaction with pharmacovigilance inspectors. 2016. Available at: http:// www.scopejointaction.eu/_assets/files/Interaction-PV-Inspectors -Survey-Report.pdf. Accessed 22 Nov 2017.

15. Ivanovic J, Schiel A, Woolley J, Shaw A, Di Girolamo M, Cicalese C, et al. SCOPE Work Package 8-lifecycle pharmacovigilance executive summary report. Available at: http://www.scopejoint action.eu/_assets/files/SCOPE-WP8-Survey-Report-2015.pdf. Accessed 22 Nov 2017.

16. Jadeja M, Inglefield P. SCOPE Work Package 4 ADR collection. An evaluation of the EU wide social media campaign to raise awareness of national spontaneous ADR reporting systems. Available at: http://www.scopejointaction.eu/_assets/files 
/2017-01-17-SCOPE-ADR-social-media-campaign-evaluation -FINAL-Mitul-Jadeja.pdf. Accessed 22 Nov 2017.

17. Jadeja M. ADR reporting awareness on social media. Uppsala reports 2017;75:20-22. Available at: https://www.who-umc.org/ media/3108/uppsalareports75web_new.pdf. Accessed 22 Nov 2017.

18. Pacurariu A, van Haren A, Berggren AL, Grundmark B, Zondag D, Harder H, et al. SCOPE Work Package 5 signal management best practice guide. June 2016. Available at: http://www.scope jointaction.eu/_assets/files/WP5-Signal-Management-Best-Pract ice-Guide(5).pdf Accessed 22 Nov 2017.

19. De Vires ST, van der Sar MJM, Coleman AM, Escudero Y, Rodriguez A, Maciá Martínez MA, et al. Safety communication tools and healthcare professionals' awareness of specific drug safety issues in Europe: a survey study. Drug Saf. 2018;41(7):713-24.

20. Alqvist-Radstad J, Andric A, Baldelli I, Buckley B, Coleman AM, Cupelli A, et al. SCOPE Work Package 6 survey report patient and consumer consultation. 2016. Available at: http://www.scope jointaction.eu/_assets/files/WP6-Patient-Consumer-ConsultationReport(1).pdf. Accessed 22 Nov 2017.

21. European Medicines Agency. The European regulatory system for medicines. A consistent approach to medicines regulation across the European Union. Available at: http://www.ema.europa.eu/ docs/en_GB/document_library/Leaflet/2014/08/WC50017167 4.pdf. Accessed 22 Nov 2017.

22. Ivanovic J, Di Girolamo M. SCOPE Work Package 8 lifecycle pharmacovigilance competency recommendations. 2016. Available at: http://www.scopejointaction.eu/_assets/files/Competency -Recommendations.pdf . Accessed 22 Nov 2017.

23. Guideline on good pharmacovigilance practices (GVP) Module IX-Signal Management (Rev 1). EMA/827661/2011 Rev 1*. Available at: http://www.ema.europa.eu/docs/en_GB/docum ent_library/Scientific_guideline/2017/10/WC500236408.pdf. Accessed 3 Jan 2018.

24. Wisniewski AFZ, Bate A, Bousquet C, Brueckner A, Candore G, Juhlin K, et al. Good signal detection practices: evidence from IMI PROTECT. Drug Saf. 2016;39:469-90.

25. Pierce C, de Vries ST, Bodin-Parssinen S, Härmark L, Tregunno $\mathrm{P}$, Lewis DL, et all. Recommendations on the Use of Mobile Applications for the Collection and Communication of Pharmaceutical Product Safety Information: Lessons from IMI WEBRADR [draft publication]. 